\title{
Optimal Control for Sustainable Consumption of Natural Resources
}

\author{
Talha Manzoor* Sergey Aseev ${ }^{* *, * * *}$ Elena Rovenskaya ${ }^{* * *}$ \\ Abubakr Muhammad* \\ * Department of Electrical Engineering, LUMS School of Science and \\ Engineering, Lahore, Pakistan \\ ** Steklov Mathematical Institute, Moscow, Russia \\ *** Advanced Systems Analysis Program, International Institute for \\ Applied Systems Analysis, Laxenburg, Austria
}

\begin{abstract}
In this paper we study optimal policies for a central planner interested in maximizing utility in an economy driven by a renewable resource. It is shown that the optimal consumption path is sustainable only when the intrinsic growth rate of the resource is greater than the social discount rate. The model is formulated as an infinite horizon optimal control problem. We deal with the mathematical details of the problem, develop a precise notion for optimality and establish the existence of optimal control at least when the condition for sustainability is met. We apply the appropriate version of the Pontryagin maximum principle and show a numerical simulation of the optimal feedback law. In the end we present the results along with physical interpretations.
\end{abstract}

\section{INTRODUCTION}

Sustainability is a natural concern in a world moving continuously towards depletion of its resources. Consumption of natural resources is driven sometimes due to the fulfillment of basic human needs and sometimes due to various growth imperatives. Whatever the case, consumption is inevitable and so, exhaustible resources such as oil, gas and other fossil fuels are sure to deplete at some point in time. In contrast to this, renewable resources such as water, fisheries and biofuels offer the possibility of sustainable consumption in which the resource supply is guaranteed not to deplete if consumption is reduced to a sufficiently low level. This minimum level of consumption may be achieved by influence of a governing body through implementation of effective policies. However, sustainability of the resource may be of little use if it comes at the cost of the essential benefits gained by consumption of that resource by a society. The aim of the work presented here is to determine the conditions under which sustainability of a renewable resource is possible without compromising the utility gained through consumption of that particular resource. We do so by posing the model as an optimal control problem.

There exist numerous studies regarding sustainability for renewable resource consumption across multiple academic disciplines. Our model for consumption compares with the one used by Roopnarine [2013], where he has modeled the Tragedy of the Commons (see Hardin [1968]) in open access renewable resources by considering the behaviors of individual agents. Here we do not explicitly consider the behaviors of individual consumers, rather we consider

\footnotetext{
ऋ This work was done during the 2013 Young Scientists Summer Program (YSSP) at IIASA, Laxenberg, Austria. T. Manzoor received a grant for YSSP participation under an IIASA-Pakistan cooperation by the Government of Pakistan.
}

the consumption pattern of the society as a whole. The assumptions of homogeneity in the lumped parameter model and existence of a central planner are part of our chosen framework. Although this framework is not uncommon (a survey is included in Beltratti et al. [1993]), the limitations induced by it must be completely understood before the deduced results are applied in practice. Considerable efforts have been made in the specification of more accurate models, for example by developing implicit models and simulations both in the context of game theory (Wiszniewska-Matyszkiel [2001], Faysse [2005]) and similar agent-based and behavioral formulations (Deadman [1999], Castillo and Saysel [2005]).

We formulate our model as an optimal control problem whose solution is a welfare-maximizing upper bound on quotas for individual consumption rates. There has been much debate over the years on what measure of social welfare to select for such optimal control problems (a philosophical treatment of the subject can be found in Sen [1985]). A common approach is the discounted utilitarianism model in which the utility gained by consumption of the resource is discounted over an infinite time horizon. The discount rate may be assumed to be fixed, time varying or uncertain (Farzin [1984], Duncan et al. [2011]). However, discounting enforces a fundamental asymmetry between present and future generations, which may not agree with at least some definitions of sustainability (see for instance, Tietenberg and Lewis [2000] and Solow [1992]). Addressing this issue, the Chichilnisky criterion developed by Chichilnisky [1996] gives a positive weight to the limiting value of the utility, in addition to the discounted utility function. This criterion is used by Beltratti et al. [1993] to develop the Green Golden Rule for sustainability in renewable resource consumption. The criteria we take here is similar to the one assumed by Aseev et al. [2010]. We take a discounted logarithmic utility function of 
the output as a measure of welfare, maximizing economic growth over an infinite time horizon. Once the optimal control problem is set up, we clearly define the notion of optimality and also investigate the existence of an optimal solution to the problem. We reformulate the problem to an equivalent one with linear dynamics and prove their equivalence. We then look for stable equilibrium points (which directly correspond to sustainable consumption in the sense described earlier) in the solutions of this problem after application of the relevant version of Pontryagin's maximum principle.

In the end we present the conditions under which sustainable consumption is possible, as guidelines for policy making. We find that in order to ensure sustainability: 1) measures must be taken in order to increase growth rate of the resource, 2) policy makers should be able to plan long-term i.e., they must plan over a large time horizon. Although one is able to infer these guidelines through intuition, the analysis we conduct here gives us an idea about how high the resource growth rate and how large the planning time horizon should be. Also, this provides an additional validation to our model and enables us to trust it as we move towards more complex scenarios for which the results might not be so intuitive.

\section{RESOURCE DYNAMICS}

Here we assume a scenario in which the resource stock dynamics have the following form

$$
\dot{R}(t)=g(R(t))-c(t) R(t),
$$

where $R(t)$ is the total resource quantity available at time $t, g(\cdot): \mathbb{R} \rightarrow \mathbb{R}$ is the growth function of the resource and $c(\cdot): \mathbb{R} \rightarrow \mathbb{R}$ represents the consumption rate of $R$ at time $t$. From the Gordon Schaefer model (Schaefer [1957]), $c(t)$ can also be interpreted as the amount of effort exerted in harvesting the resource evaluated at time $t$. The growth function $g(\cdot)$ is assumed to be logistic in nature and thus the evolution of the resource over time is described by the following differential equation

$$
\dot{R}(t)=r R(t)\left(1-\frac{R(t)}{K}\right)-c(t) R(t),
$$

where $r>0$ is the intrinsic growth rate of the resource and $K>0$ is the carrying capacity.

\section{CONTROL SCHEME AND OBJECTIVE FUNCTIONAL}

For a social planner the objective of influencing the consumption rate of the resource may be achieved through multiple ways. An indirect and relatively long term approach would be to educate the consuming population so as to alter their consumption behaviors as required. Another approach would be to control the price, which would affect the fraction of the population having access to the resource, however this may have other economic consequences especially if the resource in question plays a vital role in the economy of the consuming society. Another way would be to introduce exclusive access rights which is also one of the solutions suggested by Hardin [1968] for avoiding tragedies in open access resources. Here we are interested in obtaining upper bounds on individual consumption rates. This enables the social planner to assign quotas of consumption to individual consumers. Indeed as reported by Leal [1998], the practice of specifying quotas to control resource consumption has been found in some communities in the past as well. Thus we shall assume $c(t)$ to be our control variable.

We now describe an appropriate objective functional in order to provide some measure of welfare for the social planner to maximize. We assume that for every time instant $t \in[0, \infty)$ the output $Y(t)>0$ produced by the economy, is described by a simplified Cobb-Douglas production function

$$
Y(t)=C(c(t) R(t))^{\alpha}, \quad \text { where } \quad \alpha \in(0,1] .
$$

Here $C>0$ represents the knowledge stock, which we assume to be fixed and $\alpha$ represents the output-effort elasticity. We wish to maximize a discounted logarithmic utility function of the output, which leads us to the following objective functional (Arrow and Kruz [1970])

$$
\begin{aligned}
\tilde{J}(R(\cdot), c(\cdot)) & =\int_{0}^{\infty} \mathrm{e}^{-\rho t} \ln (Y(t)) \mathrm{d} t \rightarrow \max \\
& =\int_{0}^{\infty} \mathrm{e}^{-\rho t} \ln C(c(t) R(t))^{\alpha} \mathrm{d} t \rightarrow \max
\end{aligned}
$$

where $\rho>0$ is a discount factor. It can easily be shown that the same maximizing pair $(R(\cdot), c(\cdot))$ will be obtained by maximizing the following functional

$$
J(R(\cdot), c(\cdot))=\int_{0}^{\infty} \mathrm{e}^{-\rho t} \ln (c(t) R(t)) \mathrm{d} t \rightarrow \max .
$$

We take $J(R(\cdot), c(\cdot))$ as our final objective functional.

\section{THE OPTIMAL CONTROL PROBLEM}

Based on the discussion in Sections 2 and 3, we arrive at the following optimal control problem for renewable resource consumption

$$
\begin{aligned}
& \dot{R}(t)=r R(t)\left(1-\frac{R(t)}{K}\right)-c(t) R(t) \\
& R(0)=R_{0}>0 ; \quad c(t)>0 \\
& J(R(\cdot), c(\cdot))= \\
& \quad \int_{0}^{\infty} \mathrm{e}^{-\rho t}[\ln (c(t))+\ln (R(t))] \mathrm{d} t \rightarrow \max .
\end{aligned}
$$

Some definitions are in order here. Firstly, by an admissible control $c(\cdot):[0, \infty) \rightarrow \mathbb{R}$ in problem $(\mathrm{P} 1)$ we mean a locally bounded (bounded on any finite time interval) and measurable function which is defined on the infinite halfopen time interval $[0, \infty)$ so that

$$
\forall T>0, \quad \exists M_{T} \geq 0 \quad \text { s.t. } \quad|c(t)| \leq M_{T} .
$$

Secondly, by an admissible trajectory $R(\cdot):[0, \infty) \rightarrow \mathbb{R}$ corresponding to an admissible control $c(\cdot)$ in problem (P1), we mean a (locally) absolutely continuous function which is a solution of the differential equation in problem (P1) on an infinite time interval $[0, \infty)$, subject to the initial condition in problem (P1).

A pair $(R(\cdot), c(\cdot))$ where $c(\cdot)$ is an admissible control and $R(\cdot)$ is the corresponding admissible trajectory is called an admissible pair (or a process) in problem (P1).

The improper integral in problem $(\mathrm{P} 1)$ is defined as following 


$$
J(R(\cdot), c(\cdot))=\lim _{T \rightarrow \infty} \int_{0}^{T} \mathrm{e}^{-\rho t}[\ln (c(t))+\ln (R(t))] \mathrm{d} t,
$$

if the limit exists. To prove that the limit exists, we first need to prove some intermediate statements.

Lemma 1. There is a function $\omega:[0, \infty) \mapsto[0, \infty)$ such that $\omega(t) \rightarrow 0$ as $t \rightarrow \infty$ and for any admissible pair $(R(\cdot), c(\cdot))$ the following inequality holds true:

$\int_{T}^{T^{\prime}} e^{-\rho t}[\ln (c(t))+\ln (R(t))] \mathrm{d} t \leq \omega(T), \quad 0 \leq T<T^{\prime}$. Proof: It is easy to see that $R(t)$ is bounded from above as

$$
\sup _{t \geq 0} R(t) \leq \bar{R}=\max \left\{R_{0}, K\right\}
$$

Hence, for arbitrary $0 \leq T<T^{\prime}$ we have

$$
\begin{aligned}
& \int_{T}^{T^{\prime}} e^{-\rho t}[\ln (c(t))+\ln (R(t))] \mathrm{d} t \\
& \leq \int_{T}^{T^{\prime}} e^{-\rho t}(c(t) R(t)-1) \mathrm{d} t \\
& \leq \int_{T}^{T^{\prime}} e^{-\rho t}\left[r R(t)\left(1-\frac{R(t)}{K}\right)-\dot{R}(t)\right] \mathrm{d} t \\
& \leq \frac{r \bar{R}}{\rho}\left(e^{-\rho T}-e^{-\rho T^{\prime}}\right)+e^{-\rho T} \bar{R} \\
& \leq \omega(T)=\frac{(r+\rho) \bar{R}}{\rho} e^{-\rho T} .
\end{aligned}
$$

Lemma 2. For any admissible pair $(R(\cdot), c(\cdot))$ the limit in equality (1) exists and is either finite or equals $-\infty$.

Proof: Let us first define $J_{T}(R(\cdot), c(\cdot))$ as

$$
J_{T}(R(\cdot), c(\cdot))=\int_{0}^{T} \mathrm{e}^{-\rho t}[\ln (c(t))+\ln (R(t))] \mathrm{d} t .
$$

Next, we define a maximizing sequence $\left\{\zeta_{i}\right\}$ such that as $\zeta_{i} \rightarrow \infty$

$$
\begin{aligned}
& J_{\zeta_{i}}(R(\cdot), c(\cdot)) \rightarrow \\
& \lim _{T \rightarrow \infty} \sup _{(c(\cdot), R(\cdot))} \int_{0}^{T} \mathrm{e}^{-\rho t}[\ln (c(t))+\ln (R(t))] \mathrm{d} t .
\end{aligned}
$$

Also, we define a minimizing sequence $\left\{\tau_{i}\right\}$ such that as $\tau_{i} \rightarrow \infty$

$$
\begin{aligned}
& J_{\tau_{i}}(R(\cdot), c(\cdot)) \rightarrow \\
& \lim _{T \rightarrow \infty} \inf _{(c(\cdot), R(\cdot))} \int_{0}^{T} \mathrm{e}^{-\rho t}[\ln (c(t))+\ln (R(t))] \mathrm{d} t .
\end{aligned}
$$

Now, observing the defined functional as $\zeta_{i} \rightarrow \infty, \tau_{i} \rightarrow \infty$ and following an indexing such that $\tau_{i}<\zeta_{i}, i=1,2, \ldots$, we can write

$$
\begin{aligned}
J_{\zeta_{i}}(c(\cdot), R(\cdot)) & =J_{\tau_{i}}(c(\cdot), R(\cdot)) \\
& +\int_{\tau_{i}}^{\zeta_{i}} e^{-\rho t}[\ln (c(t))+\ln (R(t))] \mathrm{d} t \\
& \leq J_{\tau_{i}}(c(\cdot), R(\cdot))+\omega\left(\tau_{i}\right) .
\end{aligned}
$$

This implies

$$
\begin{aligned}
& \lim _{T \rightarrow \infty} \sup _{(c(\cdot), R(\cdot))} \int_{0}^{T} e^{-\rho t}[\ln (c(t))+\ln (R(t))] \mathrm{d} t \\
& \quad=\lim _{i \rightarrow \infty} J_{\zeta_{i}}(c(\cdot), R(\cdot)) \leq \lim _{i \rightarrow \infty} J_{\tau_{i}}(c(\cdot), R(\cdot)) \\
& \quad=\lim _{T \rightarrow \infty} \inf _{(c(\cdot), R(\cdot))} \int_{0}^{T} e^{-\rho t}[\ln (c(t))+\ln (R(t))] \mathrm{d} t .
\end{aligned}
$$

As far as

$$
\begin{aligned}
& \lim _{T \rightarrow \infty} \sup _{(c(\cdot), R(\cdot))} \int_{0}^{T} e^{-\rho t}[\ln (c(t))+\ln (R(t))] \mathrm{d} t \\
& \geq \lim _{T \rightarrow \infty(c(\cdot), R(\cdot))} \inf _{0}^{T} e^{-\rho t}[\ln (c(t))+\ln (R(t))] \mathrm{d} t,
\end{aligned}
$$

we get

$$
\begin{aligned}
& \lim _{T \rightarrow \infty} \sup _{(c(\cdot), R(\cdot))} \int_{0}^{T} e^{-\rho t}[\ln (c(t))+\ln (R(t))] \mathrm{d} t \\
& \quad=\lim _{T \rightarrow \infty} \inf _{(c(\cdot), R(\cdot))} \int_{0}^{T} e^{-\rho t}[\ln (c(t))+\ln (R(t))] \mathrm{d} t .
\end{aligned}
$$

Hence the limit in equality (1) exists. Due to Lemma 1 this limit is finite or equals $-\infty$.

Now we are able to define an optimal pair for problem (P1). By optimal admissible pair or optimal process in the problem (P1) we mean an admissible pair $\left(R^{*}(\cdot), c^{*}(\cdot)\right)$ such that on this pair the functional in equality (1) takes on its maximal value and the latter is finite, that is,

$$
J\left(R^{*}(\cdot), c^{*}(\cdot)\right)=\sup _{(R(\cdot), c(\cdot))} J(R(\cdot), c(\cdot))>-\infty .
$$

Here the maximum is taken over all admissible pairs $(R(\cdot), c(\cdot))$. Before we can establish the existence of such an optimal pair, we must first prove the following statement Lemma 3. For an optimal admissible pair $\left(R^{*}(\cdot), c^{*}(\cdot)\right)$ (if it exists)

$$
\begin{aligned}
J\left(R^{*}(\cdot), c^{*}(\cdot)\right) & \left.=\lim _{T \rightarrow \infty} \int_{0}^{T} e^{-\rho t}\left[\ln \left(c^{*}(t)\right)+\ln (R)(t)\right)\right] \mathrm{d} t \\
& >-\infty
\end{aligned}
$$

converges to a finite number.

Proof: From Lemma 1 we already have that for any admissible pair, $J_{T}(R(\cdot), c(\cdot))$ will either converge to $-\infty$ or a finite number. It is easy to see that the admissible control $\tilde{c}(t) \equiv r / 2, t \geq 0$, provides a finite value for the functional. Hence, if optimal admissible pair $\left(R^{*}(\cdot), c^{*}(\cdot)\right)$ exists then the corresponding value $J\left(R^{*}(\cdot), c^{*}(\cdot)\right)$ must also be finite.

\section{REDUCTION TO A PROBLEM WITH LINEAR DYNAMICS}

The state equation may be transformed into a linear one, if the following variables and parameters are introduced

$$
x(t)=\frac{1}{R(t)}, \quad u(t)=\frac{c(t)}{R(t)}, \quad A=\frac{r}{K} .
$$

Substituting this into problem $(\mathrm{P} 1)$ gives us the following new problem

$$
\begin{aligned}
& \dot{x}(t)=-r x(t)+u(t)+A, \\
& x(0)=x_{0}>0 ; \quad u(t)>0, \\
& J_{1}(x(\cdot), u(\cdot))= \\
& \quad \int_{0}^{\infty} \mathrm{e}^{-\rho t}[\ln (u(t))-2 \ln (x(t))] \mathrm{d} t \rightarrow \max ,
\end{aligned}
$$

where $x(t)$ and $u(t)$ are the auxiliary state and control variables respectively at time $t, r$ is the intrinsic growth rate of the resource, $A$ is a parameter given by equality (2), $J_{1}(x(\cdot), u(\cdot))$ is the objective functional evaluated for the functions $x(\cdot)$ and $u(\cdot)$ and $\rho$ is a positive discount factor. Note that structurally, problem (P2) is much simpler to 
evaluate than problem (P1) as it is a problem with linear dynamics. Problem (P2) is equivalent to problem (P1) in the following sense.

Lemma 4. For fixed $R_{0}$, there is a one-one correspondence between processes $(R(\cdot), c(\cdot))$ in problem $(\mathrm{P} 1)$ and $(x(\cdot), u(\cdot))$ in problem $(\mathrm{P} 2)$, where both processes are admissible in their corresponding problems. Moreover, the corresponding values of the objective functionals $J(R(\cdot), c(\cdot))$ and $J_{1}(x(\cdot), u(\cdot))$ are equal for corresponding processes where $(R(\cdot), c(\cdot))$ and $(x(\cdot), u(\cdot))$ are related by equality (2).

Proof: First we show that an admissible pair in (P1) corresponds to an admissible pair in (P2). Assume that $c(\cdot)$ is locally bounded and measurable, and consider the pair $(R(\cdot), c(\cdot))$. Now, from the state equation in $(\mathrm{P} 1)$, we have

$$
\dot{R}(t)=r R(t)\left(1-\frac{R(t)}{K}\right)-c(t) R(t) ; \quad R(0)=R_{0}>0 .
$$

We know from here that

$$
\dot{R}(t) \geq-c(t) R(t) \Longrightarrow R(t) \geq R_{0} \mathrm{e}^{-\int_{0}^{t} c(s) \mathrm{d} s},
$$

and

$$
u(t)=\frac{c(t)}{R(t)} \leq \frac{c(t)}{R_{0}} \mathrm{e}_{0}^{t} c(s) \mathrm{d} s .
$$

As $c(t)$ has been assumed to be locally bounded and measurable to begin with, the above inequality tells us that $u(t)$ must also be locally bounded and measurable. Thus an admissible pair in (P1) corresponds to an admissible pair in (P2).

Next we show that an admissible pair in (P2) corresponds to an admissible pair in (P1). Assume that the control $u(t)$ is locally bounded and measurable. The state equation in (P2) is given as

$$
\dot{x}(t)=-r x(t)+u(t)+A ; \quad x(0)=x_{0}>0 .
$$

As $u(t) \geq 0$, this equation gives us the following inequality

$$
x(t) \geq x_{0} \mathrm{e}^{-r t} \Longrightarrow c(t)=\frac{u(t)}{x(t)} \leq \frac{u(t)}{x_{0}} \mathrm{e}^{r t} .
$$

As $u(t)$ has been assumed to be locally bounded and measurable to begin with, the above inequality tells us that $c(t)$ must also be locally bounded and measurable. Thus an admissible pair in (P2) corresponds to an admissible pair in $(\mathrm{P} 1)$

Now we show that the value of the objective functionals remain the same for corresponding admissible pairs in problems (P1) and (P2). The objective functional in (P2) has already been derived by making the substitutions of equation (2) in (P1). The proof below shows that the equality of the objective functionals holds in the reverse direction as well.

$$
\begin{aligned}
& J_{1}(x(\cdot), u(\cdot))=\int_{0}^{\infty} \mathrm{e}^{-\rho t}[\ln (u(t))-2 \ln (x(t))] \mathrm{d} t \\
& =\int_{0}^{\infty} \mathrm{e}^{-\rho t}\left[\ln \left(\frac{c(t)}{R(t)}\right)-2 \ln \left(\frac{1}{R(t)}\right)\right] \mathrm{d} t \\
& =\int_{0}^{\infty} \mathrm{e}^{-\rho t}[\ln (c(t))-\ln (R(t))+2 \ln (R(t))] \mathrm{d} t \\
& =\int_{0}^{\infty} \mathrm{e}^{-\rho t}[\ln (c(t))+\ln (R(t))] \mathrm{d} t=J(R(\cdot), c(\cdot)) .
\end{aligned}
$$

This concludes the proof.
Hence problems (P1) and (P2) are equivalent, and an optimal solution in one, will correspond to an optimal solution in the other. We choose to focus on (P2) as it is simpler to solve.

\section{APPLICATION OF PONTRYAGIN'S MAXIMUM PRINCIPLE}

Here we apply the current value formulation of Pontryagin's Maximum Principle to problem (P2). A detailed account of the maximum principle for the related class of problems is given by Aseev and Veliov [2012] and Aseev et al. [2012].

Theorem 5. Let $\left(x_{*}(\cdot), u_{*}(\cdot)\right)$ be an optimal process in problem (P2). Then there exists an absolutely continuous function $\lambda(\cdot)$ (the current value adjoint variable) such that the triple $\left(x_{*}(\cdot), \lambda(\cdot), u_{*}(\cdot)\right)$ satisfies:

1) The Hamiltonian system

$$
\begin{aligned}
& \dot{x}_{*}(t)=-r x_{*}(t)+u_{*}(t)+A, \\
& \dot{\lambda}(t)=\rho \lambda(t)-\mathcal{H}_{x}\left(x_{*}(t), u_{*}(t), \lambda(t)\right),
\end{aligned}
$$

with the current value Hamiltonian

$$
\mathcal{H}(x, u, \lambda)=\ln u-2 \ln x+\lambda(-r x+u+A) .
$$

2) The maximality condition

$$
u_{*}(t)=\arg \sup _{u<0} \mathcal{H}\left(x_{*}(t), u, \lambda(t)\right) .
$$

3) The generalized transversality condition

$$
\lambda(t)=\mathrm{e}^{\rho t} Z_{*}(t) \int_{t}^{\infty} \phi(s) \mathrm{d} s,
$$

where $\phi(s)$ is given by

$$
\phi(s)=\mathrm{e}^{-\rho s}\left[Z_{*}(s)\right]^{-1} \frac{\partial}{\partial x}\left(\ln \left(u_{*}(s)\right)-2 \ln \left(x_{*}(s)\right)\right),
$$

and $Z_{*}(t)$ is the normalized fundamental matrix of the system

$$
\dot{z}(t)=-\frac{\partial}{\partial x}(-r x(t)+u(t)+A) z(t) .
$$

\section{ANALYSIS OF THE HAMILTONIAN SYSTEM}

From the maximality condition 2) in Theorem 5, we can find $u_{*}(t)$. Maximization of the current value Hamiltonian $\mathcal{H}\left(x_{*}(t), u, \lambda(t)\right)$ leads us to the following

$$
\left.\frac{\partial \mathcal{H}}{\partial u}\right|_{u=u_{*}(t)}=\frac{1}{u_{*}(t)}+\lambda(t)=0 \Longrightarrow u_{*}(t)=-\frac{1}{\lambda(t)} \text {. }
$$

To confirm that the expression for $u_{*}(t)$ indeed maximizes the current value Hamiltonian we evaluate the 2 nd partial derivative

$$
\left.\frac{\partial^{2} \mathcal{H}}{\partial u^{2}}\right|_{u=u_{*}(t)}=-\frac{1}{u_{*}^{2}(t)}<0,
$$

which shows that $u_{*}(t)$ indeed maximizes the current value Hamiltonian. Replacing this into the Hamiltonian system shown in Theorem 5 we get the following system

$$
\begin{aligned}
& \dot{x}(t)=-r x(t)-\frac{1}{\lambda(t)}+A=F(x, \lambda), \\
& \dot{\lambda}(t)=(\rho+r) \lambda(t)+\frac{2}{x(t)}=G(x, \lambda) .
\end{aligned}
$$

We are interested in an equilibrium analysis of the Hamiltonian system (4), as stable equilibrium points of this 
system correspond directly to sustainable consumption. It is easy to see that a unique equilibrium point exists, given as

$$
\left(\bar{x}_{*}, \bar{\lambda}\right)=\left(\frac{2 A}{r-\rho}, \frac{\rho-r}{A(r+\rho)}\right) .
$$

From here we see that the quadrant of the phase plane in which the equilibrium point lies depends upon the values of $r$ and $\rho$. From equality (3), we can see that in order to have a physically realizable equilibrium $\left(\bar{x}_{*}>0, \bar{u}_{*}>0\right)$, the point $\left(\bar{x}_{*}, \bar{\lambda}\right)$ must lie in the 4 th quadrant. From here we see that this happens only when $r>\rho$.

The stability of the equilibrium can be observed by analyzing the eigenvalues of the linearized system. The linearized system about $\left(\bar{x}_{*}, \bar{\lambda}\right)$ and its eigenvalues are given by

$$
\left[\begin{array}{cc}
-r & \frac{A^{2}(r+\rho)^{2}}{(\rho-r)^{2}} \\
-\frac{(r-\rho)^{2}}{2 A^{2}} & r+\rho
\end{array}\right], \quad \sigma_{1,2}=\frac{\rho}{2} \pm \frac{1}{2} \sqrt{2 r^{2}-\rho^{2}} .
$$

By observing the expression above, we can see that the eigenvalues may either be real numbers with different sign, real numbers both with positive sign or complex numbers both with positive real parts. We see that in order for them to be real numbers with different sign, the following must hold true

$$
\sqrt{2 r^{2}-\rho^{2}}>\rho \Longrightarrow r>\rho .
$$

Thus if $r>\rho$, then $\left(\bar{x}_{*}, \bar{\lambda}\right)$ lies in the 3rd quadrant with eigenvalues which are real numbers having different sign. This corresponds to a saddle point (both for the linearized and non-linear systems) which is sustainable if the state is made to follow along the 2 stable trajectories. This is illustrated in Figure 1.

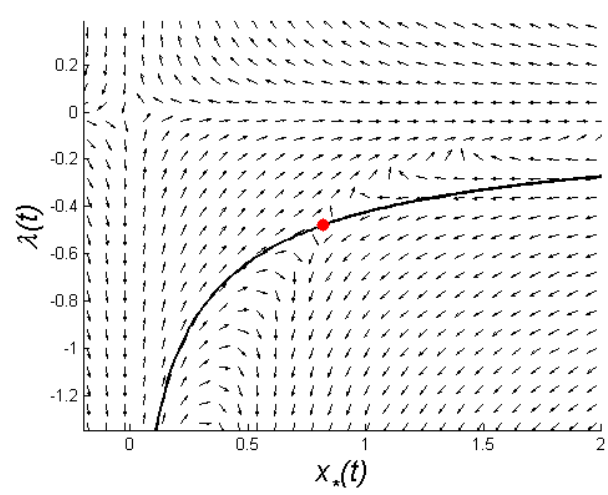

Figure 1. Direction field of the Hamiltonian system (4). The equilibrium point $\left(\bar{x}_{*}, \bar{\lambda}\right)$ has been marked in red and the stable trajectories are represented by the solid curve in black. Here $r=5, \rho=0.1$ and $A=2$.

\subsection{The optimal feedback law}

From Figure 1 it is apparent that the optimal trajectories will lie along the stable trajectories. Let us call the trajectory corresponding to $x<\bar{x}$ as $\lambda_{-}(x)$ and the one corresponding to $x>\bar{x}$ as $\lambda_{+}(x)$. Using this information and equality (3) we can get the optimal feedback law as

$$
u_{*}(x)= \begin{cases}-\frac{1}{\lambda_{-}(x)}, & \text { if } x<\bar{x}, \\ -\frac{A(r+\rho)}{\rho-r}, & \text { if } x=\bar{x} \\ -\frac{1}{\lambda_{+}(x)}, & \text { if } x>\bar{x} .\end{cases}
$$

which means that in order to find the optimal feedback, we must be able to determine the trajectories $\lambda_{-}(x)$ and $\lambda_{+}(x)$. We can do this by determining the solution of the following differential equation passing through $\left(\bar{x}_{*}, \bar{\lambda}\right)$

$$
\frac{d \lambda}{d x}=\frac{d \lambda}{d t} \times \frac{d t}{d x}=\frac{G(x, \lambda)}{F(x, \lambda)}=\frac{\lambda((\rho+r) \lambda x+2)}{x(-r \lambda x-1+A \lambda)} .
$$

An analytical solution to this nonlinear differential equation is difficult to obtain. However it is possible to solve numerically. An example of the numerically obtained solution can be seen in Figure 2 where the auxiliary variables have been translated into the variables of problem (P1).

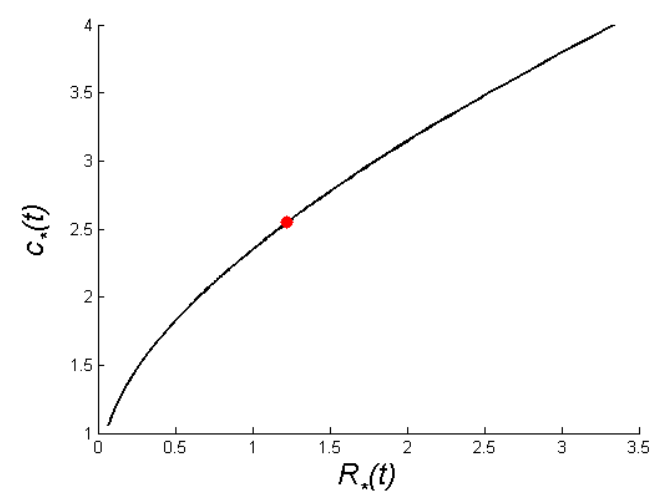

Figure 2. A graphical representation of the optimal feedback for the same system depicted in Figure 1 . The equilibrium point $\left(\bar{R}_{*}, \bar{c}_{*}\right)$ has been marked in red.

\section{EXISTENCE OF OPTIMAL CONTROL}

Pontryagin's maximum principle describes a necessary condition for the optimal control (if it exists). However, the existence of the optimal control must be proved separately, which is what we do here. Note that the argument given here is valid only when $r>\rho$. When $r \leq \rho$ either an optimal control does not exist, or sustainability is not possible, and so it is not of our interest. Consider the following optimal control problem

$$
\begin{aligned}
& \dot{x}(t)=f(x(t), u(t)), \\
& x(0)=x_{0}>0, \quad B_{1} \leq u(t) \leq B_{2}, \\
& J(R(\cdot), c(\cdot))=\int_{0}^{\infty} \mathrm{e}^{-\rho t} g(x(t), u(t)) \mathrm{d} t \rightarrow \max .
\end{aligned}
$$

Here the control $u(t)$ is bounded for all times. From the standard existence result (see for example Aseev et al. [2012]), we know that an optimal solution for such problems exists. By this and from the results obtained for problem (P2) and Figures 1 and 2, we can see that the optimal control will be bounded as follows

$$
u_{*}(t) \in\left[\frac{1}{L}, L\right]
$$

where the number $L$ will be dependent only on the initial condition $x_{0}$ and will increase as $x_{0}$ increases. But as $x_{0}$ is bounded (through $R_{0}$ ), there will come a point where $L$ will become uniform and independent of $x_{0}$. Thus for a sufficiently large value of $L$, problem (P2) can be considered equivalent to problem (P3). As the existence of optimal control for (P3) is already established, this guarantees the existence of optimal control in problem (P2) and consequently in problem (P1). 


\section{POLICY GUIDELINES FOR SUSTAINABILITY}

Based on the analysis conducted in Section 7 we conclude that in order to ensure the possibility of sustainable consumption, the following condition must hold true

$$
r>\rho,
$$

where $r$ is the intrinsic growth rate of the resource, and $\rho$ is the positive discount factor. This condition implies the following guidelines for policy makers

- Increase resource growth rate: Sometimes the growth rate of the resource is so low that sustainability might simply not be possible. Of course even in this case a sufficiently low consumption rate can stabilize the resource quantity at a certain level, but it will not be sustainable in the sense of maximizing the selected measure for welfare (Section 3). It may also not always be possible to increase the growth rate of the resource as this is an intrinsic property. But we must realize when it is possible e.g. in wind and solar farms by improving equipment technology, in fisheries by providing an encouraging environment for reproduction, in hydrology by channeling rainwater and water from melting glaciers more effectively, and so on.

- Plan long term: Decreasing the discount factor $\rho$ corresponds to giving more weight to future benefits. If this factor is kept large, the only optimal solution will be to consume maximum resource in the present and not worry about the future. In other words, if the planning horizon is too small, then the future is so unimportant that it is more efficient to consume as much as possible today. It is important to note here that choosing a small discount factor is not just a matter of choosing a parameter for an equation. In order to enable policy makers to consider a large planning horizon, there must be stability in the society. In a society struck with chaos and instability, there is a large uncertainty on what the future will be. In this case it is not sensible to choose a large planning horizon and thus, not possible to ensure sustainability.

\section{CONCLUSION}

In this paper we have dealt with an infinite horizon optimal control problem for renewable resource consumption. We have proved that for our model, the optimal consumption path is sustainable only when the intrinsic growth rate of the resource is greater than the discount rate. Although one might think beforehand that a high growth rate and long term planning strategy are prerequisites for sustainable resource consumption, it might not be clear as to exactly how high the growth rate or exactly how long term the planning should be. The condition given by (5) gives us some idea about the answer to these questions. In the process we have also taken a step towards solving a class of infinite horizon optimal control problems with unbounded control, which may have applications other than the problem of natural resource consumption.

\section{REFERENCES}

Kenneth J Arrow and Mordecai Kruz. Public investment, the rate of return, and optimal fiscal policy, volume 1. Routledge, 1970.

Sergei M Aseev, Konstantin O Besov, and Arkadii V Kryazhimskii. Infinite-horizon optimal control problems in economics. Russian Mathematical Surveys, 67(2):195, 2012.

Sergey Aseev, Konstantin Besov, and Serguei Kaniovski. Optimal endogenous growth with exhaustible resources. IIASA Interim Report IR-10-011, 2010.

SM Aseev and VM Veliov. Needle variations in infinitehorizon optimal control. Research Report 2012-04, ORCOS, Vienna University of Technology, Vienna, 2012.

Andrea Beltratti, Graciela Chichilnisky, and Geoffrey Heal. Sustainable growth and the green golden rule. Technical report, National Bureau of Economic Research, 1993.

Daniel Castillo and Ali Kerem Saysel. Simulation of common pool resource field experiments: a behavioral model of collective action. Ecological economics, 55(3): 420-436, 2005.

Graciela Chichilnisky. An axiomatic approach to sustainable development. Social choice and welfare, 13(2):231257, 1996.

Peter J Deadman. Modelling individual behaviour and group performance in an intelligent agent-based simulation of the tragedy of the commons. Journal of Environmental Management, 56(3):159-172, 1999.

Stephen Duncan, Cameron Hepburn, and Antonis Papachristodoulou. Optimal harvesting of fish stocks under a time-varying discount rate. Journal of theoretical biology, 269(1):166-173, 2011.

$\mathrm{Y}$ Hossein Farzin. The effect of the discount rate on depletion of exhaustible resources. The Journal of Political Economy, pages 841-851, 1984.

Nicolas Faysse. Coping with the tragedy of the commons: Game structure and design of rules. Journal of economic surveys, 19(2):239-261, 2005.

Garrett Hardin. The tragedy of the commons. New York, 1968.

Donald R Leal. Community-run fisheries: Avoiding the tragedy of the commons. Population and Environment, 19(3):225-245, 1998.

Peter Roopnarine. Ecology and the tragedy of the commons. Sustainability, 5(2):749-773, 2013.

Milner B Schaefer. Some considerations of population dynamics and economics in relation to the management of the commercial marine fisheries. Journal of the Fisheries Board of Canada, 14(5):669-681, 1957.

Amartya Sen. Well-being, agency and freedom: the dewey lectures 1984. The Journal of Philosophy, 82(4):169221, 1985.

Robert M Solow. An almost practical step toward sustainability. Resources for the Future, 1992.

Thomas H Tietenberg and Lynne Lewis. Environmental and natural resource economics. Addison-Wesley Reading, MA, 2000.

Agnieszka Wiszniewska-Matyszkiel. The tragedy of the commons modelled by large games. In Advances in Dynamic Games and Applications, pages 323-345. Springer, 2001. 